\title{
Axial-vector currents and $\tau$ mesonic decays
}

\author{
Bing An $\mathrm{Li}^{\mathrm{a} *}$

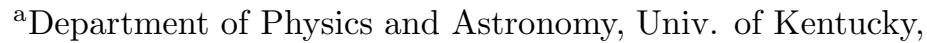 \\ Lexington, Kentucky
}

A general expression of the axial-vector current is presented, in which both the effects of the chiral symmetry breaking and the spontaneous chiral symmetry breaking are included. A new resonance formula of the axial-vector meson is derived and in the limit of $q^{2} \rightarrow 0$ this formula doesn't go back to the "chiral limit". The studies show that the dominance of the axial-vector meson is associated with the satisfaction of PCAC. The dominance of pion exchange is companied by the strong anomaly of PCAC.

\section{INTRODUCTION}

The $\tau$ mesonic decays have been studied by many authors[1]. Chiral symmetry play an important role in studying $\tau$ mesonic decays. In the theory of $\tau$ mesonic decays there are three parts: vector currents; axial-vector currents; meson vertices. The vector currents are treated by VMD. In the chiral limit, we have

$$
\begin{aligned}
\mathcal{L}^{V}= & \frac{g_{W}}{4 f_{\rho}} \cos \theta_{C}\left\{-\frac{1}{2}\left(\partial_{\mu} A_{\nu}^{i}-\partial_{\nu} A_{\mu}^{i}\right)\left(\partial_{\mu} \rho_{\nu}^{i}-\partial_{\nu} \rho_{\mu}^{i}\right)\right. \\
& \left.+A_{\mu}^{i} j^{i \mu}\right\}
\end{aligned}
$$

$j_{\mu}^{i}$ is derived by the substitution

$\rho_{\mu}^{i} \rightarrow \frac{g_{W}}{4 f_{\rho}} \cos \theta_{C} A_{\mu}^{i}$

in the vertices involving $\rho$ mesons.

There are three terms in the axial-vector current. It is known for a long time that $a_{1}$ meson is the chiral partner of the $\rho$ meson, therefore, there should be a term which is similar with VMD, axial-vector meson dominance. However, $a_{1}$ is much heavier than $\rho$ and the mass difference is resulted in spontaneous chiral symmetry breaking. The second term is related to the mass difference of $a_{1}$ and $\rho$ meson. The third term comes from the coupling between the pion and W-boson. Combining these three terms, the general expression of the axial-vector current is obtained

$\mathcal{L}^{A}=-\frac{g_{W}}{4 f_{a}} \cos \theta_{C}\left\{-\frac{1}{2}\left(\partial_{\mu} A_{\nu}^{i}-\partial_{\nu} A_{\mu}^{i}\right)\right.$

${ }^{*}$ Work partially supported by the Department of Energy under grant DE-91ER75661.

$$
\begin{aligned}
& \left.\left(\partial_{\mu} a_{\nu}^{i}-\partial_{\nu} a_{\mu}^{i}\right)+A^{i \mu} j_{\mu}^{i W}\right\} \\
& -\frac{g_{W}}{4} \cos \theta_{C} \Delta m^{2} f_{a} A_{\mu}^{i} a^{i \mu} \\
& -\frac{g_{W}}{4} \cos \theta_{C} f_{\pi} A_{\mu}^{i} \partial^{\mu} \pi^{i},
\end{aligned}
$$

where $j_{\mu}^{i W}$ is determined by

$a_{\mu}^{i} \rightarrow \frac{g_{W}}{4 f_{a}} \cos \theta A_{\mu}^{i}$.

There are two parameters $f_{a}$ and $\Delta m^{2}$. Using Weinberg's first sum rule and the mass difference between $\rho$ and $a_{1}$ meson, the two parameters are determined

$f_{a}^{2}=f_{\rho}^{2}\left(1-\frac{f_{\pi}^{2} f_{\rho}^{2}}{m_{\rho}^{2}}\right) \frac{m_{a}^{2}}{m_{\rho}^{2}}, \quad \Delta m^{2}=f_{\pi}^{2}\left(1-\frac{f_{\pi}^{2} f_{\rho}^{2}}{m_{\rho}^{2}}\right)^{-1}$.

An effective chiral theory of pseudoscalar, vector, and axial-vector mesons has been proposed[2], in which the meson fields are simulated by quark operators. For example,

$\rho_{\mu}^{i}=-\frac{1}{g_{\rho} m_{\rho}^{2}} \bar{\psi} \tau_{i} \gamma_{\mu} \psi$.

The realization of quark operator forms of meson fields is done by constructing a proper Lagrangian

$$
\begin{aligned}
\mathcal{L}= & \bar{\psi}(x)\left(i \gamma \cdot \partial+\gamma \cdot v+\gamma \cdot a \gamma_{5}-m u(x)\right) \psi(x) \\
& -\bar{\psi}(x) M \psi(x) \\
& +\frac{1}{2} m_{0}^{2}\left(\rho_{i}^{\mu} \rho_{\mu i}+\omega^{\mu} \omega_{\mu}+a_{i}^{\mu} a_{\mu i}+f^{\mu} f_{\mu}\right)
\end{aligned}
$$

where $a_{\mu}=\tau_{i} a_{\mu}^{i}+f_{\mu}, v_{\mu}=\tau_{i} \rho_{\mu}^{i}+\omega_{\mu}$, and

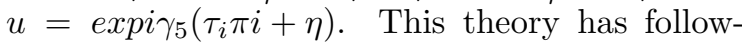


ing features: chiral symmetry; spontaneous chiral symmetry breaking; quark condensate; Vector Meson Dominance(VMD) is a natural result; Weinberg sum rule and KSFR sum rule are satisfied; Wess-Zumino-Witten Lagrangain is the leading term of the imaginary part of the effective Lagrangian; constituent quark mass is determined; large $N_{C}$ expansion is revealed and all loop diagrams of mesons are at higher order; two parameters of Chiral Perturbation Theory are determined in the chiral limit; there are five parameters: three current quark masses, a cut-off, and a universal coupling constant which is chosen to be $g=0.39$; theoretical results of masses, decay widths, $\pi \pi$ scattering agree with data well. This theory is $Q C D$ inspired, self-consistent, and phenomenologically successful. The cut-off $\Lambda$ is determined to be $1.6 \mathrm{GeV}$ which makes the theory suitable for the studies of $\tau$ mesonic decays. In this theory they are determined: $\Gamma_{\rho}=142 \mathrm{MeV}$ and $m_{a}=1.20 \mathrm{GeV}$.

We have applied this theory to study $\tau$ mesonic decays[3]. The VMD is a natural result of this theory and the expression of the axial-vector current is derived. The meson vertices of the $\tau$ decays are found from the effective Lagrangaian of mesons [2,3]. Therefore, this theory provides a unified study for $\tau$ mesonic decays. There are additional features in the studies of $\tau$ mesonic decays [3]:

1. The vertex VPP depends on momentum. For example, $f_{\rho \pi \pi}$ derived from the effective Lagrangian[3] is no longer a constant, but a function of $q^{2}$

$f_{\rho \pi \pi}\left(q^{2}\right)=\frac{2}{g}\left\{1+\frac{q^{2}}{2 \pi^{2} f_{\pi}^{2}}\left[\left(1-\frac{2 c}{g}\right)^{2}-4 \pi^{2} c^{2}\right]\right\}$,

where $c=\frac{f_{\pi}^{2}}{2 g m_{\rho}^{2}}$. The radius of the charged pion is determined to be

$$
\begin{aligned}
& r_{\pi}^{2}=\frac{6}{m_{\rho}^{2}}+\frac{3}{\pi^{2} f_{\pi}^{2}}\left\{\left(1-\frac{2 c}{g}\right)^{2}-4 \pi^{2} c^{2}\right\} \\
& =0.447 \mathrm{fm}^{2}
\end{aligned}
$$

which is in excellent agreement with data.
2. The vertex AVP depends on momentum.

$$
\begin{aligned}
& \mathcal{L}^{a_{1} \rho \pi}=\epsilon_{i j k}\left\{A a_{\mu}^{i} \rho^{j \mu} \pi^{k}-B a_{\mu}^{i} \rho_{\nu}^{j} \partial^{\mu \nu} \pi^{k}\right\}, \\
& A=\frac{2}{f_{\pi}} g f_{a}\left\{\frac{m_{a}^{2}}{g^{2} f_{a}^{2}}-m_{\rho}^{2}\right. \\
& +p^{2}\left[\frac{2 c}{g}+\frac{3}{4 \pi^{2} g^{2}}\left(1-\frac{2 c}{g}\right)\right] \\
& \left.+q^{2}\left[\frac{1}{2 \pi^{2} g^{2}}-\frac{2 c}{g}-\frac{3}{4 \pi^{2} g^{2}}\left(1-\frac{2 c}{g}\right)\right]\right\} \\
& B=-\frac{2}{f_{\pi}} g f_{a} \frac{1}{2 \pi^{2} g^{2}}\left(1-\frac{2 c}{g}\right),
\end{aligned}
$$

where $\mathrm{p}$ and $\mathrm{q}$ are momentum of $\rho$ and $a_{1}$ respectively.

3. The resonance formula of the axial-vector meson takes a new form

$\frac{\Delta m^{2} f_{a}^{2}-m_{a}^{2}+i \sqrt{q^{2}} \Gamma_{a}\left(q^{2}\right)}{q^{2}-m_{a}^{2}+i \sqrt{q^{2}} \Gamma_{a}\left(q^{2}\right)}$,

where $\Delta m^{2} f_{a}^{2}$ is related to the spontaneous chiral symmetry breaking. In the limit of $q^{2} \rightarrow 0$ this formula doesn't go to the "chiral limit".

4. The normalization constants of the vector and the axial-vector mesons are different.

The tree diagrams are at the leading order in large $N_{C}$ expansion. All the calculations are done at the tree level.

\section{2. $a_{1}$ dominance in $\tau \rightarrow \pi \pi \pi \nu$}

The vertices $a_{1} \rho \pi, \rho \pi \pi, W \rho \pi$ contribute to this decay mode. This theory explains the existence of a $\rho$ resonance in the final state of this decay mode. There is a cancelation between the diagrams. After taking the cancelation into account, we obtain

$$
\begin{gathered}
<\rho^{0} \pi^{-}\left|\bar{\psi} \tau_{-} \gamma_{\mu} \gamma_{5} \psi\right| 0>=\frac{i}{\sqrt{4 \omega E}}\left(\frac{q_{\mu} q_{\nu}}{q^{2}}-g_{\mu \nu}\right) \\
\left(A g_{\nu \lambda}+B k_{\nu} k_{\lambda}\right) \epsilon_{\sigma}^{* \nu} \\
\frac{g^{2} f_{a} m_{\rho}^{2}-i f_{a}^{-1} \sqrt{q^{2}} \Gamma_{a}\left(q^{2}\right)}{q^{2}-m_{a}^{2}+i \sqrt{q^{2}} \Gamma_{a}\left(q^{2}\right)}
\end{gathered}
$$


The $a_{1}$ dominance in $\tau \rightarrow \rho \pi \nu$ is revealed and the dominance is caused by the cancelation which leads to the axial-vector current conservation in the chiral limit. The branching ratios are computed to be

$$
B\left(\tau \rightarrow \pi^{+} \pi^{-} \pi^{-} \nu\right)=B\left(\tau \rightarrow \pi^{-} \pi^{0} \pi^{0} \nu\right)=6.3 \% .
$$

The decay width is determined to be

$\Gamma_{a}=386 \mathrm{MeV}$.

The ratio of the decay amplitudes of the $a_{1}$ meson, $d / s$, is calculated to be -0.098 . These results are in reasonable agreement with the data.

\section{3. $a_{1}$ dominance in $\tau \rightarrow f_{1} \pi \nu$}

$f_{1}$ meson is the chiral partner of $\omega$ meson[2] and the mass formula of $f_{1}$ meson is derived in Ref.[2]

$$
\left(1-\frac{1}{2 \pi^{2} g^{2}}\right) m_{f_{1}}^{2}=6 m^{2}+m_{\omega}^{2}, \quad m_{f_{1}}=1.21 G e V .
$$

The vertex of $f_{1}(1285) a_{1} \pi$

$\mathcal{L}^{f_{1} a_{1} \pi}=\frac{1}{\pi^{2} f_{\pi}} \frac{1}{g^{2}}\left(1-\frac{1}{2 \pi^{2} g^{2}}\right)^{-1} \varepsilon^{\mu \nu \alpha \beta} f_{\mu} \partial_{\nu} \pi^{i} \partial_{\alpha} a_{\beta}^{i}$

is from the Wess-Zumino-Witten anomaly and it is used to calculate the decay rate of this decay mode. The narrow width of the decay $f_{1} \rightarrow \rho \pi \pi$ is revealed from this vertex[2]. $a_{1}$ is dominant in $\tau \rightarrow f_{1} \pi \nu$.

$B\left(\tau \rightarrow f_{1} \pi \nu\right)=2.91 \times 10^{-4}$.

The data is $(6.7 \pm 1.4 \pm 2.2) \times 10^{-4}(\mathrm{CLEO}$ recent measurement).

\section{Effective Lagrangian of $\Delta s=1$ weak in- teractions}

Using the $K^{*}$ fields to substitute the $\rho$ fields in Eq.(1), the VMD of $\Delta s=1$ is obtained. In Ref.[2] the chiral partner of $K^{*}(892)$ meson, the $K_{1}$ meson, is coupled to

$\bar{\psi} \lambda^{a} \gamma_{\mu} \gamma_{5} \psi$.

The mass of this $K_{1}$ meson is derived as

$$
\left(1-\frac{1}{2 \pi^{2} g^{2}}\right) m_{K_{1}}^{2}=6 m^{2}+m_{K^{*}}^{2},
$$

$m_{K_{1}}=1.32 \mathrm{GeV}$.

It is lower than the mass of $K_{1}(1400)$ and greater than $K_{1}(1270)$ 's mass. The widths of three decay modes $\left(K_{1} \rightarrow K^{*} \pi, K \rho, K \omega\right)$ are calculated[2]. It is found that $K^{*} \pi$ channel is dominant, however, $B(K \rho)$ is about $11 \%$. The data shows that the branching ratio of $K_{1}(1400)$ decaying into $K \rho$ is very small. Therefore, the meson coupled to the quark axial-vector current is not a pure $K_{1}(1400)$ state, instead, it is a mixture of the two $K_{1}$ mesons. It is $K_{a}$.

$K_{a}=\cos \theta K_{1}(1400)+\sin \theta K_{1}(1270)$,

$K_{b}=-\sin \theta K_{1}(1400)+\cos \theta K_{1}(1270)$.

In this theory the amplitude of $\tau \rightarrow K_{a} \nu$ is from the tree diagrams and at $O\left(N_{C}\right)$ [2]. The production of $K_{b}$ in $\tau$ decay is through loop diagrams of mesons which is at $O(1)$ in large $N_{c}$ expansion[2]. This theory predicts a small branching ratio for $K_{b}$ production in $\tau$ decays. The axialvector current of $\Delta s=1$ is obtained by using $K_{a}$ to substitute $a_{1}$ in Eq.(3).

5. $\tau \rightarrow K^{*} \pi \nu$

Both the vector and axial-vector currents contribute to this decay. The vertex $\pi K^{*} \bar{K}^{*}$ contributes to the vector part and it is from the WZW anomaly[3]. The vector matrix element is obtained

$$
\begin{gathered}
<\bar{K}^{* 0} \pi^{-}\left|\bar{\psi} \lambda_{+} \gamma_{\mu} \psi\right| 0>=\frac{-1}{\sqrt{4 \omega E}} \frac{1}{\sqrt{2}} \frac{N_{C}}{\pi^{2} g^{2} f_{\pi}} \\
\frac{m_{K^{*}}^{2}-i \sqrt{q^{2}} \Gamma_{K^{*}}\left(q^{2}\right)}{q^{2}-m_{K^{*}}^{2}+i \sqrt{q^{2}} \Gamma_{K^{*}}\left(q^{2}\right)} \varepsilon^{\mu \nu \alpha \beta} k_{\nu} p_{\alpha} \epsilon_{\beta}^{* \sigma},
\end{gathered}
$$

where $\mathrm{p}$ and $\mathrm{k}$ are momentum of $K^{*}$ and pion respectively, $q=k+p$.

The axial-vector matrix element is obtained by using the vertices: $K_{a} K^{*} \pi, K K^{*} \pi$. In the chiral limit, the expression of the matrix element of the axial-vector current is derived

$$
\begin{aligned}
& <\bar{K}^{* 0} \pi^{-}\left|\bar{\psi} \lambda_{+} \gamma_{\mu} \gamma_{5} \psi\right| 0>= \\
& \quad \frac{i}{\sqrt{4 \omega E}} \frac{1}{\sqrt{2}}\left(\frac{q_{\mu} q_{\nu}}{q^{2}}-g_{\mu \nu}\right) \epsilon_{\sigma}^{* \lambda}
\end{aligned}
$$




$$
\begin{aligned}
& \left\{\frac{g^{2} f_{a} m_{K^{*}}^{2}-i q f_{a}^{-1} \Gamma_{K_{1}(1400)}\left(q^{2}\right)}{q^{2}-m_{K_{1}(1400)}^{2}+i q \Gamma_{K_{1}(1400)}\left(q^{2}\right)}\right. \\
& \cos \theta\left(A_{K_{1}(1400)}\left(q^{2}\right)_{K^{*}} g^{\nu \lambda}+B_{K_{1}(1400)} k^{\nu} k^{\lambda}\right) \\
& +\frac{g^{2} f_{a} m_{K^{*}}^{2}-i q f_{a}^{-1} \Gamma_{K_{1}(1270)}\left(q^{2}\right)}{q^{2}-m_{K_{1}(1270)}^{2}+i q \Gamma_{K_{1}(1270)}\left(q^{2}\right)} \\
& \sin \theta\left(A_{K_{1}(1270)}\left(q^{2}\right)_{K^{*}} g^{\nu \lambda}\right. \\
& \left.\left.+B_{K_{1}(1270)} k^{\nu} k^{\lambda}\right)\right\}
\end{aligned}
$$

The amplitudes $A_{K_{1}(1400)}, B_{K_{1}(1400)}, A_{K_{1}(1270)}$, and $B_{K_{1}(1270)}$ are determined by the decay widths of $K_{1}(1400)$ and $K_{1}(1270)$.

$K_{1}(1400)=\cos \theta K_{a}-\sin \theta K_{b}$,

$K_{1}(1270)=\sin \theta K_{a}+\cos \theta K_{b}$.

The vertex of $K_{1} V P$ is found from Ref.[2]

$$
\begin{gathered}
\mathcal{L}^{K_{1} V P}=f_{a b c}\left\{A K_{1 \mu}^{a} V^{b \mu} P^{c}\right. \\
\left.-B K^{a \mu} V^{b \nu} \partial_{\mu} \partial_{\nu} P^{c}\right\} .
\end{gathered}
$$

The amplitude $A_{K_{a}}^{K^{*}}$ is determined to be

$$
\begin{aligned}
A\left(q^{2}\right)_{K_{a}}^{K^{*}} & =\frac{2}{f_{\pi}} g f_{a}\left\{\frac{m_{K_{a}}^{2}}{g^{2} f_{a}^{2}}-m_{K^{*}}^{2}\right. \\
& +m_{K^{*}}^{2}\left[\frac{2 c}{g}+\frac{3}{4 \pi^{2} g^{2}}\left(1-\frac{2 c}{g}\right)\right] \\
& \left.+q^{2}\left[\frac{1}{2 \pi^{2} g^{2}}-\frac{2 c}{g}-\frac{3}{4 \pi^{2} g^{2}}\left(1-\frac{2 c}{g}\right)\right]\right\} .
\end{aligned}
$$

$B_{K_{a}}$ is the same as B. The amplitudes $A_{K_{b}}^{K^{*}}$ and $B_{K_{b}}^{K^{*}}$ are unknown and we take them as parameters. Both $K_{1}(1400)$ and $K_{1}(1270)$ decay to $K \rho$ and $K \omega$. Using the $S U(3)$ coefficients, for both $K_{1}$, it is determined

$$
B(K \omega)=\frac{1}{3} B(K \rho) \text {. }
$$

This relation agrees with data reasonably well. For the $K \rho$ decay mode $A_{K_{b}}^{\rho}$ and $B_{K_{b}}^{\rho}$ are other two parameters. In the decays of the two $K_{1}$ mesons the momentum of pion or kaon is low, therefore, the decay widths are insensitive to the amplitude B. We take

$B_{K_{b}}^{K^{*}}=B_{K_{b}}^{\rho} \equiv B_{b}$.

The decay width of the $K_{1}$ meson is derived

$\Gamma_{K_{1}}=\frac{k}{32 \pi} \frac{1}{\sqrt{q^{2}} m_{K_{1}}}\left\{\left(3+\frac{k^{2}}{m_{v}^{2}}\right) A^{2}\left(q^{2}\right)\right.$

$$
\left.-A\left(q^{2}\right) B\left(q^{2}+m_{v}^{2}\right) \frac{k^{2}}{m_{v}^{2}}+\frac{q^{2}}{m_{v}^{2}} k^{4} B^{2}\right\},
$$

where $q^{2}=m_{K_{1}}^{2}, v=K^{*}, \rho, \mathrm{k}$ is the momentum of pion or kaon

$k=\left\{\frac{1}{4 m_{K_{1}}^{2}}\left(m_{K_{1}}^{2}+m_{v}^{2}-m_{P}^{2}\right)^{2}-4 m_{v}^{2}\right\}^{\frac{1}{2}}$,

$m_{P}$ is the mass of pion or kaon.

we choose the parameters as

$$
\begin{aligned}
& \theta=30^{0}, A_{b}^{K^{*}}=-4.5 \mathrm{GeV}, \quad A_{b}^{\rho}=5.0 \mathrm{GeV}, \\
& B_{b}=0.8 \mathrm{GeV}^{-1},
\end{aligned}
$$

from which the decay widths are obtained

$$
\begin{gathered}
\Gamma\left(K_{1}(1400) \rightarrow K^{*} \pi\right)=159 \mathrm{MeV}, \\
\Gamma\left(K_{1}(1400) \rightarrow K \rho\right)=10.5 \mathrm{MeV}, \\
\Gamma\left(K_{1}(1270) \rightarrow K^{*} \pi\right)=12.4 \mathrm{MeV}, \\
\Gamma\left(K_{1}(1270) \rightarrow K \rho\right)=26.8 \mathrm{MeV} .
\end{gathered}
$$

Using the two matrix elements $(7,8)$, the distribution of the decay rate is derived

$$
\begin{aligned}
& \frac{d \Gamma}{d q^{2}}\left(\tau^{-} \rightarrow \bar{K}^{* 0} \pi^{-} \nu\right)=\frac{G^{2}}{(2 \pi)^{3}} \frac{\sin ^{2} \theta_{C}}{128 m_{\tau}^{3} q^{4}}\left(m_{\tau}^{2}-q^{2}\right)^{2} \\
& \left(m_{\tau}^{2}+2 q^{2}\right)\left\{\left(q^{2}+m_{K^{*}}^{2}-m_{\pi}^{2}\right)^{2}-4 q^{2} m_{K^{*}}^{2}\right\}^{\frac{1}{2}} \\
& \left\{\frac{6}{\pi^{4} g^{2} f_{\pi}^{2}} \frac{m_{K^{*}}^{4}+q^{2} \Gamma_{K^{*}}^{2}\left(q^{2}\right)}{\left(q^{2}-m_{K^{*}}^{2}\right)^{2}+q^{2} \Gamma_{K^{*}}^{2}\left(q^{2}\right)}\right. \\
& \quad\left[(p \cdot q)^{2}-q^{2} m_{K^{*}}^{2}\right] \\
& \quad+|A|^{2}\left[1+\frac{1}{12 m_{K^{*}}^{2} q^{2}}\left(q^{2}-m_{K^{*}}^{2}\right)^{2}\right] \\
& \quad-\left(B A^{*}+B^{*} A\right) \frac{\left(q^{2}+m_{K^{*}}^{2}\right)}{24 m_{K^{*}}^{2} q^{2}}\left(q^{2}-m_{K^{*}}^{2}\right)^{2} \\
& \left.\quad+\frac{|B|^{2}}{48 m_{K^{*}}^{2} q^{2}}\left(q^{2}-m_{K^{*}}^{2}\right)^{4}\right\} .
\end{aligned}
$$

where $\mathrm{p}$ is the momentum of $K^{*}, q^{2}$ is the invariant mass squared of $K^{*} \pi$, and

$$
\begin{aligned}
& A=\frac{g^{2} f_{a} m_{K^{*}}^{2}-i \sqrt{q^{2}} f_{a}^{-1} \Gamma_{K_{1}(1400)}}{q^{2}-m_{K_{1}(1400)}^{2}+i \sqrt{q^{2}} \Gamma_{K_{1}(1400)}} \cos \theta A_{K_{1}(1400)}^{K^{*}} \\
& +\frac{g^{2} f_{a} m_{K^{*}}^{2}-i \sqrt{q^{2}} f_{a}^{-1} \Gamma_{K_{1}(1270)}}{q^{2}-m_{K_{1}(1270)}^{2}+i \sqrt{q^{2}} \Gamma_{K_{1}(1270)}} \sin \theta A_{K_{1}(1270)}^{K^{*}}, \\
& B=\frac{g^{2} f_{a} m_{K^{*}}^{2}-i \sqrt{q^{2}} f_{a}^{-1} \Gamma_{K_{1}(1400)}}{q^{2}-m_{K_{1}(1400)}^{2}+i \sqrt{q^{2}} \Gamma_{K_{1}(1400)}} \cos \theta B_{K_{1}(1400)}^{K^{*}}
\end{aligned}
$$


$+\frac{g^{2} f_{a} m_{K^{*}}^{2}-i \sqrt{q^{2}} f_{a}^{-1} \Gamma_{K_{1}(1270)}}{q^{2}-m_{K_{1}(1270)}^{2}+i \sqrt{q^{2}} \Gamma_{K_{1}(1270)}} \sin \theta B_{K_{1}(1270)}^{K^{*}}$,

where

$$
\begin{gathered}
A_{K_{1}(1400)}^{K^{*}}=\cos \theta A_{a}^{K^{*}}-\sin \theta A_{b}^{K^{*}} \\
B_{K_{1}(1400)}^{K^{*}}=\cos \theta B_{a}-\sin \theta B_{b}, \\
A_{K_{1}(1270)}^{K^{*}}=\sin \theta A_{a}^{K^{*}}+\cos \theta A_{b}^{K^{*}} \\
B_{K_{1}(1270)}^{K^{*}}=\sin \theta B_{a}+\cos \theta B_{b} .
\end{gathered}
$$

In the range of $q^{2}$, the main decay channels of $K^{*}$ are $K \pi$ and $K \eta$. The decay width of $K^{*}$ is derived

$$
\begin{gathered}
\Gamma\left(q^{2}\right)_{K^{*}}=\frac{f_{\rho \pi \pi}^{2}\left(q^{2}\right)}{8 \pi} \frac{k^{3}}{\sqrt{q^{2}} m_{K^{*}}} \\
+\cos ^{2} 20^{0} \frac{f_{\rho \pi \pi}^{2}\left(q^{2}\right)}{8 \pi} \frac{k^{\prime 3}}{\sqrt{q^{2}} m_{K^{*}}}, \\
k=\left\{\frac{1}{4 q^{2}}\left(q^{2}+m_{K}^{2}-m_{\pi}^{2}\right)^{2}-m_{K}^{2}\right\}^{\frac{1}{2}}, \\
k^{\prime}=\left\{\frac{1}{4 q^{2}}\left(q^{2}+m_{K}^{2}-m_{\eta}^{2}\right)^{2}-m_{K}^{2}\right\}^{\frac{1}{2}} .
\end{gathered}
$$

In $\Gamma_{K_{1}}\left(q^{2}\right)$ the decay modes $K^{*} \pi, K \rho$ and $K \omega$ are included

$$
\begin{aligned}
\Gamma\left(q^{2}\right)_{K_{1}} & =\frac{k}{32 \pi} \frac{1}{\sqrt{q^{2}} m_{K_{1}}}\left\{\left(3+\frac{k^{2}}{m_{K^{*}}^{2}}\right) A^{2}\left(q^{2}\right)_{K^{*}}\right. \\
- & \left.A\left(q^{2}\right)_{K^{*}} B\left(q^{2}+m_{K^{*}}^{2}\right) \frac{k^{2}}{m_{K^{*}}^{2}}+\frac{q^{2}}{m_{K^{*}}^{2}} k^{4} B^{2}\right\} \\
+ & \frac{4}{3} \frac{k^{\prime}}{32 \pi} \frac{1}{\sqrt{q^{2} m_{K_{1}}}}\left\{\left(3+\frac{k^{2}}{m_{\rho}^{2}}\right) A^{2}\left(q^{2}\right)\right. \\
- & \left.A\left(q^{2}\right) B\left(q^{2}+m_{\rho}^{2}\right) \frac{k^{2}}{m_{\rho}^{2}}+\frac{q^{2}}{m_{\rho}^{2}} k^{4} B^{2}\right\}, \\
k & =\left\{\frac{1}{4 q^{2}}\left(q^{2}+m_{K^{*}}^{2}-m_{\pi}^{2}\right)^{2}-m_{K^{*}}^{2}\right\}^{\frac{1}{2}}, \\
k^{\prime} & =\left\{\frac{1}{4 q^{2}}\left(q^{2}+m_{K}^{2}-m_{\rho}^{2}\right)^{2}-m_{K}^{2}\right\}^{\frac{1}{2}} .
\end{aligned}
$$

For $K_{1}(1270), \Gamma\left(K_{1}(1270) \rightarrow K_{0}^{*}(1430) \pi\right)=$ $25.2 \mathrm{MeV}$ is included. The branching ratio is calculated

$$
B\left(\tau^{-} \rightarrow \bar{K}^{* 0} \pi^{-} \nu\right)=0.23 \%,
$$

The contribution of the vector current is about $7.4 \%$. Therefore, $K_{a}$ is dominant in this decay.
The data are

$0.38 \pm 0.11 \pm 0.13 \%$ (CLEO)

$0.25 \pm 0.10 \pm 0.05 \%$ (ARGUS)

There is another decay channel $\tau^{-} \rightarrow K^{*-} \pi^{0} \nu$ whose branching ratio is one half of $B\left(\tau^{-} \rightarrow\right.$ $\bar{K}^{* 0} \pi^{-} \nu$. The total branching ratio is

$B\left(\tau^{-} \rightarrow \bar{K} \pi \nu\right)=0.35 \%$,

The width is about $230 \mathrm{MeV}$.

\section{6. $\tau \rightarrow K \rho \nu$ and $K \omega \nu$}

It is the same as $\tau \rightarrow K^{*} \pi \nu, K_{a}$ dominates the decay $\tau \rightarrow K \rho \nu$. Both the vector and axialvector currents contribute to this decay mode. The matrix element of the vector current, < $\bar{K}^{0} \rho^{-}\left|\bar{\psi} \lambda_{+} \gamma_{\mu} \psi\right| 0>$ is determined by the abnormal vertex $\mathcal{L}^{K^{*} K \rho}(47)$. The axial-vector matrix element $<\bar{K}^{0} \rho^{-}\left|\bar{\psi} \lambda_{+} \gamma_{\mu} \gamma_{5} \psi\right| 0>$ is obtained by substituting

$K^{*} \rightarrow \rho, \quad K \rightarrow \pi$

in Eq.(8). Using the same substitutions in Eq.(15), the distribution of the decay rate of $\tau \rightarrow K \rho \nu$ is found. The branching ratio of $\tau \rightarrow K \rho \nu$ (two modes $\bar{K}^{0} \rho^{-}$and $K^{-} \rho^{0}$ ) is computed to be

$B=0.75 \times 10^{-3}$.

It is about $18 \%$ of $\tau \rightarrow K \pi \pi \nu$. The vector current makes $8 \%$ contribution. The DELPHI has reported that $\tau \rightarrow K^{*} \pi \nu$ is dominant the decay $\tau \rightarrow K \pi \pi \nu$ and $K \rho \nu$ decay mode has not been observed. The recent ALEPH's results are the $K^{*} \pi$ dominance and a branching ratio of $30 \pm 11 \%$ for the $K \rho$ mode.

Due to the $S U(3)$ coefficient we expect

$B(\tau \rightarrow K \omega \nu)=\frac{1}{3} B(\tau \rightarrow K \rho \nu)$.

7. $\tau \rightarrow K^{*} \eta$

There are vector and axial-vector parts in this decay. The calculation of the decay rate is similar to the decay of $\tau \rightarrow K^{*} \pi \nu$. The vertices $\mathcal{L}^{K^{*} \bar{K}^{*} \eta}$ and $\mathcal{L}^{W K^{*} \eta}$ via the Lagrangian $L^{V s}$ contribute to the vector part and the vertices $\mathcal{L}^{K_{1} K^{*} \eta}, \mathcal{L}^{K K^{*} \eta}$, and $\mathcal{L}^{W K^{*} \eta}$ via $L^{A s}$ take the responsibility for 
the axial-vector part. The vertex $\mathcal{L}^{K^{*} \bar{K}^{*} \eta}$ comes from anomaly. Using the same method deriving the vertices $\eta v v$ (in Ref.[2]), it is found

$$
\begin{gathered}
\mathcal{L}^{K^{*} \bar{K}^{*} \eta}=-\frac{3 a}{2 \pi^{2} g^{2} f_{\pi}} d_{a b 8} \varepsilon^{\mu \nu \alpha \beta} \eta \partial_{\mu} K_{\nu}^{a} \partial_{\alpha} K_{\beta}^{b} \\
-\frac{3 b}{2 \pi^{2} g^{2} f_{\pi}} \varepsilon^{\mu \nu \alpha \beta} \eta \partial_{\mu} K_{\nu}^{a} \partial_{\alpha} K_{\beta}^{a},
\end{gathered}
$$

where $\mathrm{a}$ and $\mathrm{b}$ are the octet and singlet component of $\eta$ respectively, $a=\cos \theta, b=\sqrt{\frac{2}{3}} \cos \theta$, and $\theta=-20^{\circ}$. Due to the cancelation between the two components the vector matrix element is very small and can be ignored.

The vertices $\mathcal{L}^{K_{1} K^{*} \eta}$ and $\mathcal{L}^{K K^{*} \eta}$ contribute to the axial-vector matrix element and they are derived from the effective Lagrangian presented in Ref.[2].

$$
\begin{aligned}
& \mathcal{L}^{K_{1} K^{*} \eta}=a f_{a b 8}\left\{A\left(q^{2}\right)_{K^{*}} K_{\mu}^{a} K^{b \nu} \eta\right. \\
& \left.-B K_{\mu}^{a} K_{\nu}^{b} \partial^{\mu \nu} \eta\right\}, \\
& \mathcal{L}^{K^{*} K \eta}=a f_{K^{*} K \eta} f_{a b 8} K_{\mu}^{a}\left(K^{b} \partial^{\mu} \eta-\eta \partial^{\mu} K^{b}\right),
\end{aligned}
$$

where $f_{K^{*} K \eta}$ is the same as $f_{\rho \pi \pi}$ in the limit of $m_{q}=0$. The decay width is similar to the one of $\tau \rightarrow K^{*} \pi \nu$

$$
\begin{aligned}
& \frac{d \Gamma}{d q^{2}}\left(\tau^{-} \rightarrow K^{*-} \eta \nu\right)= \\
& \quad \frac{G^{2}}{(2 \pi)^{3}} \cos ^{2} 20^{0} \frac{\sin ^{2} \theta_{C}}{64 m_{\tau}^{3} q^{4}}\left(m_{\tau}^{2}-q^{2}\right)^{2} \\
& \quad\left(m_{\tau}^{2}+2 q^{2}\right)\left\{\left(q^{2}+m_{K^{*}}^{2}-m_{\pi}^{2}\right)^{2}-4 q^{2} m_{K^{*}}^{2}\right\}^{\frac{1}{2}} \\
& \quad\left\{\frac{1}{2 \pi^{4} g^{2} f_{\pi}^{2}} \frac{m_{K^{*}}^{4}+q^{2} \Gamma_{K^{*}}^{2}\left(q^{2}\right)}{\left(q^{2}-m_{K^{*}}^{2}\right)^{2}+q^{2} \Gamma_{K^{*}}^{2}\left(q^{2}\right)}\right\} \\
& \quad\left[(p \cdot q)^{2}-q^{2} m_{K^{*}}^{2}\right] \\
& \quad+\frac{3}{4}\left\{|A|^{2}\left[1+\frac{1}{12 m_{K^{*}}^{2} q^{2}}\left(q^{2}-m_{K^{*}}^{2}\right)^{2}\right]\right. \\
& \quad-\left(B A^{*}+B^{*} A\right) \frac{\left(q^{2}+m_{K^{*}}^{2}\right)}{24 m_{K^{*}}^{2} q^{2}}\left(q^{2}-m_{K^{*}}^{2}\right)^{2} \\
& \left.\left.\left.\quad+\frac{|B|^{2}}{48 m_{K^{*}}^{2} q^{2}}\left(q^{2}-m_{K^{*}}^{2}\right)^{4}\right]\right\}\right\} .
\end{aligned}
$$

The branching ratio is computed to be

$B=1.05 \times 10^{-4}$.

The contribution of the vector current is $3.3 \%$ and the axial-vector current is dominant.

\section{Strong anomaly of PCAC}

$$
\tau \rightarrow \omega \rho \nu
$$

Only the axial-vector currents contribute to $\tau \rightarrow$ $\omega \rho \nu$. At the tree level $\mathcal{L}^{\omega \rho \pi}$ is the only vertex involved in this decay channel

$\mathcal{L}^{\omega \rho \pi}=-\frac{N_{C}}{\pi^{2} g^{2} f_{\pi}} \varepsilon^{\mu \nu \alpha \beta} \partial_{\mu} \omega_{\nu} \partial_{\alpha} \rho_{\beta}^{i} \pi^{i}$.

This vertex has been tested by $\tau \rightarrow \omega \pi \nu, \pi^{0} \rightarrow$ $2 \gamma, \omega \rightarrow \pi \gamma, \rho \rightarrow \pi \gamma$, and $\omega \rightarrow 3 \pi$.

The matrix element of the axial-vector current is obtained

$$
\begin{gathered}
<\omega \rho^{-}\left|\bar{\psi} \tau_{+} \gamma_{\mu} \gamma_{5} \psi\right| 0>=-\frac{i}{\sqrt{4 E_{1} E_{2}}} \frac{N_{C}}{\pi^{2} g^{2}} \frac{q_{\mu}}{q^{2}} \varepsilon^{\lambda \nu \alpha \beta} \\
p_{1 \lambda} p_{2 \nu} \epsilon_{\alpha}^{*}\left(p_{1}\right) \epsilon_{\beta}^{*}\left(p_{2}\right) .
\end{gathered}
$$

In the limit $m_{q}=0$, the axial-vector current is not conserved in this process. The pion exchange dominates this decay.

The decay width is

$$
\begin{aligned}
\Gamma= & \frac{G^{2}}{128 m_{\tau}} \frac{\cos ^{2} \theta_{C}}{(2 \pi)^{3}} \frac{9}{\pi^{4} g^{4}} \int_{q_{m i n}^{2}}^{m_{\tau}^{2}} d q^{2} \frac{1}{q^{6}}\left(m_{\tau}^{2}-q^{2}\right)^{2} \\
& \int_{4 m_{\pi}^{2}}^{\left(\sqrt{q^{2}}-m_{\omega}\right)^{2}} d k^{2}\left[\left(q^{2}+m_{\omega}^{2}-k^{2}\right)^{2}-4 q^{2} m_{\omega}^{2}\right]^{\frac{3}{2}} \\
& \frac{1}{\pi} \frac{\sqrt{k^{2}} \Gamma_{\rho}\left(k^{2}\right)}{\left(k^{2}-m_{\rho}^{2}\right)^{2}+k^{2} \Gamma_{\rho}^{2}\left(k^{2}\right)}
\end{aligned}
$$

where $q_{\min }^{2}=2 m_{\pi} m_{\tau}+\frac{m_{\tau} m_{\omega}^{2}}{m_{\tau}-2 m_{\pi}}, k^{2}$ is the invariant mass of the two pions and

$\Gamma_{\rho}\left(k^{2}\right)=\frac{f_{\rho \pi \pi}^{2}\left(k^{2}\right)}{48 \pi} \frac{k^{2}}{m_{\rho}}\left(1-4 \frac{m_{\pi}^{2}}{k^{2}}\right)^{3}$.

The branching ratio is computed to be

$B=0.16 \times 10^{-4}$.

The nonconservation of the quark axial-vector currents found in the matrix elements shows that there is anomaly in the PCAC. Taking one abnormal term as an example of the strong anomaly of the PCAC, the PCAC with strong anomaly is written as

$\partial^{\mu} \bar{\psi} \tau_{i} \gamma_{\mu} \gamma_{5} \psi=-m_{\pi}^{2} f_{\pi} \pi_{i}+\frac{N_{C}}{\pi^{2} g^{2}} \varepsilon^{\mu \nu \alpha \beta} \partial_{\mu} \omega_{\nu} \partial_{\alpha} \rho_{\beta}^{i}$ 
The abnormal term originates in the WZW anomaly.

On the other hand, using the VMD we derive

$$
\begin{aligned}
\partial^{\mu} \bar{\psi} \tau_{i} \gamma_{\mu} \gamma_{5} \psi=-m_{\pi}^{2} f_{\pi} \pi_{i}+\frac{N_{C}}{\pi^{2} g^{2}} \varepsilon^{\mu \nu \alpha \beta} \partial_{\mu} \omega_{\nu} \partial_{\alpha} \rho_{\beta}^{i} \\
\quad+\frac{\alpha}{4 \pi} \varepsilon^{\mu \nu \alpha \beta} F_{\mu \nu} F_{\alpha \beta} \delta_{3 i} \\
\quad+\frac{e}{4 \pi^{2} g} \varepsilon^{\mu \nu \alpha \beta} F_{\mu \nu} \partial_{\alpha} \rho_{\beta}^{i} \\
\quad+\frac{3 e}{4 \pi^{2} g} \varepsilon^{\mu \nu \alpha \beta} \partial_{\mu} \omega_{\nu} F_{\alpha \beta} \delta_{3 i} .
\end{aligned}
$$

The background of this process comes from $\tau \rightarrow \omega(\pi \pi)_{\text {non } \rho} \nu$. Only the axial-vector currents contribute to $\tau \rightarrow \omega(\pi \pi)_{n o n \rho} \nu$. There are two kinds of vertices involved in this decay channel. These vertices are derived from the effective chiral theory of mesons[2] in the chiral limit.

1. The vertices $\omega \pi \pi \pi$ and $\omega a_{1} \pi \pi$ are

$$
\begin{gathered}
\mathcal{L}^{\omega \pi \pi \pi}=\frac{2}{\pi^{2} g f_{\pi}^{3}}\left(1-\frac{6 c}{g}+\frac{6 c^{2}}{g^{2}}\right) \varepsilon^{\mu \nu \alpha \beta} \\
\epsilon_{i j k} \omega_{\mu} \partial_{\nu} \pi_{i} \partial_{\alpha} \pi_{j} \partial_{\beta} \pi_{k} \\
\mathcal{L}^{\omega a_{1} \pi \pi}=-\frac{6}{\pi^{2} g^{2} f_{\pi}^{2}}\left(1-\frac{1}{2 \pi^{2} g^{2}}\right)^{-\frac{1}{2}} \\
\left(1-\frac{2 c}{g}\right) \varepsilon^{\mu \nu \alpha \beta} \epsilon_{i j k} \partial_{\nu} \omega_{\mu} a_{\alpha}^{i} \partial_{\beta} \pi_{k} .
\end{gathered}
$$

These two vertices are from the WZW anomaly[2]. Using these vertices and $\mathcal{L}^{A}$, the matrix element of the axial-vector current is obtained

$$
\begin{aligned}
<\omega \pi^{0} \pi^{-}\left|\bar{\psi} \tau_{+} \gamma_{\mu} \gamma_{5} \psi\right| 0>(1)=\frac{i}{\sqrt{8 \omega_{1} \omega_{2} E}} \\
\quad \frac{6}{\pi^{2} g f_{\pi}^{2}} \varepsilon^{\nu \lambda \alpha \beta} \epsilon_{\lambda}^{*} p_{\alpha}\left(k_{2}-k_{1}\right)_{\beta} \\
\left\{\left(\frac{q_{\mu} q_{\nu}}{q^{2}}-g_{\mu \nu}\right)\left(1-\frac{2 c}{g}\right)\right. \\
\frac{g^{2} f_{a}^{2} m_{\rho}^{2}-i \sqrt{q^{2}} \Gamma_{a}\left(q^{2}\right)}{q^{2}-m_{a}^{2}+i \sqrt{q^{2}} \Gamma_{a}\left(q^{2}\right)} \\
\left.+\frac{q_{\mu} q_{\nu}}{q^{2}} \frac{2 c}{g}\left(1-\frac{2 c}{g}\right)\right\},
\end{aligned}
$$

where $k_{1}, k_{2}$, and $\mathrm{p}$ are momentum of $\pi^{0}$, $\pi^{-}$, and $\omega$ respectively, $q=k_{1}+k_{2}+p$. The equation shows that the matrix element obtained from the WZW anomaly is not conserved in the limit of $m_{q}=0$. The pion exchange is dominant in the term which violates the conservation of the quark axialvector current in the chiral limit.

2. The second kind of vertices are vertices $a_{1} \rho \pi, \rho \pi \pi, W \rho \pi$ and $\omega \rho \pi$. The vertex $\omega \rho \pi$ is from the WZW anomaly. Using the vertex $\mathcal{L}^{a_{1} \rho \pi}$, the decay width of $a_{1}$ meson is derived

$$
\begin{array}{r}
\Gamma_{a}\left(q^{2}\right)=\frac{k}{12 \pi m_{a} \sqrt{q^{2}}}\left\{\left(3+\frac{k^{2}}{m_{\rho}^{2}}\right) A^{2}\right. \\
\left.-\frac{k^{2}}{m_{\rho}^{2}}\left(q^{2}+m_{\rho}^{2}\right) A B+\frac{q^{2}}{m_{\rho}^{2}} k^{4} B^{2}\right\},
\end{array}
$$

where

$$
k=\left\{\frac{1}{4 q^{2}}\left(q^{2}+m_{\rho}^{2}-m_{\pi}^{2}\right)^{2}-m_{\rho}^{2}\right\}^{\frac{1}{2}} .
$$

Using $\mathcal{L}^{A}$ and the vertices, the second part of the matrix element of the axial-vector current is obtained

$$
\begin{aligned}
&<\omega \pi^{0} \pi^{-}\left|\bar{\psi} \tau_{+} \gamma_{\mu} \gamma_{5} \psi\right| 0>^{(2)} \\
&=\frac{i}{\sqrt{8 \omega_{1} \omega_{2} E}}\left(\frac{q_{\mu} q_{\nu}}{q^{2}}-g_{\mu \nu}\right) \\
& \frac{3}{\pi^{2} g^{2} f_{\pi}} \frac{g^{2} f_{a} m_{\rho}^{2}-i f_{a}^{-1} q \Gamma_{a}\left(q^{2}\right)}{q^{2}-m_{a}^{2}+i q \Gamma_{a}\left(q^{2}\right)} \\
& \varepsilon^{\nu \lambda \alpha \beta} \epsilon_{\lambda}^{*} p_{\alpha}\left\{\frac{A\left(k^{2}\right) k_{2 \beta}}{k^{2}-m_{\rho}^{2}+i \sqrt{k^{2}} \Gamma_{\rho}\left(k^{2}\right)}\right. \\
&\left.-\frac{A\left(k^{\prime 2}\right) k_{1 \beta}}{k^{\prime 2}-m_{\rho}^{2}+i \sqrt{k^{\prime 2}} \Gamma_{\rho}\left(k^{\prime 2}\right)}\right\} \\
&+\frac{i}{\sqrt{8 \omega_{1} \omega_{2} E}}\left(\frac{q_{\mu} q_{\nu}}{q^{2}}-g_{\mu \nu}\right) \frac{3}{\pi^{2} g^{2} f_{\pi}} \\
& \frac{g^{2} f_{a} m_{\rho}^{2}-i q f_{a}^{-1} \Gamma_{a}\left(q^{2}\right)}{q^{2}-m_{a}^{2}+i q \Gamma_{a}\left(q^{2}\right)} \\
& \varepsilon^{\sigma \lambda \alpha \beta} \epsilon_{\sigma}^{*} p_{\lambda} k_{2 \alpha} k_{1 \beta}(-B) \\
&\left\{\frac{k_{2 \nu}}{k^{\prime 2}-m_{\rho}^{2}+i \sqrt{k^{\prime 2}} \Gamma_{\rho}\left(k^{\prime 2}\right)}\right. \\
&\left.+\frac{k_{1 \nu}}{k^{2}-m_{\rho}^{2}+i \sqrt{k^{2}} \Gamma_{\rho}\left(k^{2}\right)}\right\},
\end{aligned}
$$


where $k_{1}, k_{2}, \mathrm{p}$ are momentum of $\pi^{0}, \pi^{-}$, and $\omega$ mesons respectively, $q=p+k_{1}+k_{2}$, $k=q-k_{1}, k^{\prime}=q-k_{2}, A\left(k^{2}\right)$ and $A\left(k^{\prime 2}\right)$ are defined by Eq.(16) by taking $p^{2}=k^{2}, k^{2}$ respectively. This part of the matrix element observes the axial-vector current conservation in the limit $m_{q}=0$ and there is $a_{1}$ dominance.

The theory makes explicit prediction on this decay The branching ratio is computed to be

$B=0.37 \%$.

The data are $0.39 \pm 0.04 \pm 0.04 \%$ (CLEO) and $0.41 \pm 0.08 \pm 0.06 \%(\mathrm{ALEPH})$.

\section{Summary of other results}

The decay $\tau \rightarrow K^{*} K \nu$ is calculated[3]. Both the vector and axial-vector currents contribute to this process. For the vector part only the $\rho$ meson is taken into account. The vertex is $K^{*} K \rho$ from WZW anomaly. The vector current makes $92.5 \%$ contribution. The peak of the spectrum is at $1.5 \mathrm{GeV}$. The theoretical result of the decay rate is compatible with the data.

The $K^{*}$ meson is dominant the decay $\tau \rightarrow$ $\eta K \nu[3]$. The vertex $\eta K^{*} K$ has a form factor which is the same as $f_{\rho \pi \pi}\left(q^{2}\right)$. We obtain[3] $B=2.22 \times 10^{-4}$.

The decays, $\tau \rightarrow \eta \pi \pi \nu$ and $\eta^{\prime} \pi \pi \nu$ are calculated in terms the theory[3]. Besides the vertices $\eta \rho \rho$ and $\eta^{\prime} \rho \rho$, there are contact terms. It is found that the contributions of the contact terms are negligible. The theoretical results are

$B(\tau \rightarrow \eta 2 \pi \nu)=1.9 \times 10^{-3}$,

$B\left(\tau \rightarrow \eta^{\cdot} 2 \pi \nu\right)=0.44 \times 10^{-5}$.

\section{Conclusions}

1. So far, the theory agrees with data reasonably well.

2. In two flavor cases the $a_{1}$ dominance is associated with PCAC.

3. The dominance of pion exchange is associated with the strong anomaly of PCAC.
4. $K_{a}$ dominates the axial-vector currents of $\Delta s=1$.

5. The momentum dependeces of the vertices VPP and AVP are very important in understanding the $\tau$ mesonic decays.

6. The new formula of the resonance of the axial-vector meson doesn't have the "chiral limit" at low $q^{2}$.

\section{ACKNOWLEDGEMENTS}

The author would like to thank E.Braaten, J.Smith, and R.Stroynowsky for discussion.

\section{REFERENCES}

1. F. J. Gilman and S .H .Rhie, Phys.Rev.D31, 1066(1985); S. I. Eidelman and V. N. Ivanchenko, Proc. of the Third Workshop on Tau Physics, p.131, Montreux, Switzerland, 19-22 September 1994, ed. by L.Rolandi; Y.S.Tsai, Phys.Rev.D4, 2821(1971); R.Fischer, J.Wess, and F.Wagner, Z.Phys. C3,313(1980); G.Aubrecht II, N.Chahrouri, and K.Slanec, Phys.Rev.D24, 1318(1981); E.Braaten, R.J.Oakes, and S.M.Tse, Inter.Jour.Modern Phys., 5,2737(1990); R.Decker, E.Mirkes, R.Sauer and Z.Was, Z.Phys., C58,445(1993); M.Finkemeier and E.Mirkes, Z.Phys., C69, 243(1996); G.Kramer and W.F.Palmer, Z.Phys., C25,195(1984) and ibid, $\quad 39,423(1988)$; A.Pich, Phys.Lett., 196,561(1987); R.Decker, Z.Phys., C36,487(1987); E.Braaten, R.oakes, and

S.Z.Tse, Phys.Rev., D36,2187(1987); R.Decker and E.Mirkes, Phys.Rev., D47,4012(1993).

2. B.A.Li, Phys.Rev., D52, 5165(1995), 5184(1995).

3. B.A.Li, "Theory of $\tau$ mesonic decays", hep$\mathrm{ph} / 9606402$ and " $\tau$ mesonic decays and strong anomaly of PCAC", hep-ph/9607354. 